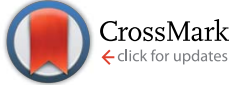

Cite this: RSC Adv., 2016, 6, 80049

\title{
Robust superhydrophilic patterning of superhydrophobic ormosil surfaces for high- throughput on-chip screening applications $\dagger$
}

\author{
Pinar Beyazkilic, ${ }^{\text {ab }}$ Urandelger Tuvshindorj, ${ }^{a b}$ Adem Yildirim, $^{\text {ab }}$ Caglar Elbuken $^{\text {ab }}$ \\ and Mehmet Bayindir*abc
}

\begin{abstract}
This article describes a facile method for the preparation of two-dimensionally patterned superhydrophobic hybrid coatings with controlled wettability. Superhydrophobic coatings were deposited from nanostructured organically modified silica (ormosil) colloids that were synthesized via a simple sol-gel method. On the defined areas of the superhydrophobic ormosil coatings, stable wetted micropatterns were produced using Ultraviolet/Ozone (UV/O) treatment which modifies the surface chemistry from hydrophobic to hydrophilic without changing the surface morphology. The degree of wettability can be precisely controlled depending on the UV/O exposure duration; extremely wetted spots with water contact angle (WCA) of nearly $0^{\circ}$ can be obtained. Furthermore, we demonstrated high-throughput biomolecular adsorption and mixing using the superhydrophilic patterns. The proposed superhydrophilic-patterned nanostructured ormosil surfaces with their simple preparation, robust and controlled wettability as well as adaptability on flexible substrates, hold great potential for biomedical and chemical on-chip analysis.
\end{abstract}

Received 3rd August 2016
Accepted 16th August 2016

DOI: $10.1039 / c 6 r a 19669 a$

www.rsc.org/advances printing, ${ }^{6,26-28}$ direct laser writing ${ }^{29}$ and photo-catalytic decomposition. ${ }^{5,30-35}$ Alternatively, UV/O treatment offers a very simple and cost-effective way for superhydrophilic patterning. Mano and co-workers prepared superhydrophilic-patterned superhydrophobic polymer surfaces with well-controlled wettability using UV/O treatment. ${ }^{7,15}$ However, contact angle increases on polymer surfaces over time after the treatment (hydrophobic recovery effect) due to the flexibility and reorganization of the bonds within the polymer chains. ${ }^{36-38}$

Here, we report a facile method for the generation of very stable superhydrophilic/superhydrophobic patterned surfaces. Superhydrophobic surfaces were prepared from nanostructured ormosil nanoparticles synthesized via sol-gel method where hydrophobic surface chemistry and high surface roughness were combined in one-pot approach. In order to create isolated super-wetted/non-wetted patterns, selected regions of the superhydrophobic surfaces were simply treated with UV/O using a shadow mask on their top. UV/O modified the surface two-dimensionally by switching its chemistry from hydrophobic methyl groups to hydrophilic silanol groups without changing the surface morphology. Super-wetted patterns maintained their wetting performance during several months of storage suggesting their suitability for practical applications. We demonstrate that degree of wettability of the hydrophilic patterns can be precisely controlled depending on the UV/O exposure duration. Superhydrophilic spots with WCA of nearly $0^{\circ}$ were formed while unmodified regions had WCA close to $170^{\circ}$. One can rapidly form droplets arrays on the patterned 
surfaces by dipping them into aqueous media or can allot microdroplets individually to separate micron-sized superhydrophilic spots. Extreme wettability difference between the untreated and UV/O-treated regions was exploited for selective adsorption and mixing of water-dispersible substances on the hydrophilic spots. In addition, superhydrophilic-superhydrophobic patterns were intact under bending impacts owing to the robustness and flexibility of ormosil coatings.

\section{Experimental section}

\subsection{Materials}

Methyltrimethoxysilane (MTMS), oxalic acid, ammonium hydroxide $(25 \%)$ and glucose were purchased from Merck (Germany). Methanol was purchased from Carlo-Erba (Italy). Fluorescein isothiocyanate-bovine serum albumin conjugate (FITC-BSA) was purchased from Sigma-Aldrich (US). All chemicals were used as received. Deionized (DI) water $(18.2 \mathrm{M} \Omega \mathrm{cm}$ at $25^{\circ} \mathrm{C}$ ) was obtained using a Millipore Milli-Q water purification system (Billerica, US).

\subsection{Preparation of superhydrophobic ormosil coatings}

Ormosil superhydrophobic coatings were prepared via two step sol-gel reaction. First, $1 \mathrm{~mL}$ of MTMS was dissolved in $9.74 \mathrm{~mL}$ of methanol. Following $15 \mathrm{~min}$-stirring of the solution, $0.5 \mathrm{~mL}$ of $1 \mathrm{mM}$ oxalic acid solution was added dropwise and the solution was gently stirred for $30 \mathrm{~min}$. Then, the mixture was left to hydrolyse the organosilane precursor completely for $24 \mathrm{~h}$ at room temperature. After the hydrolysis step, $0.19 \mathrm{~mL}$ of water and $0.42 \mathrm{~mL}$ of ammonia solution (25\%) were added gently as the catalyst of the reaction and the mixture was stirred for 15 min. The resultant mixture was left for 2 days at $25{ }^{\circ} \mathrm{C}$ for complete gelation and aging. After aging the gel network, $9 \mathrm{~mL}$ of methanol was added followed by sonication using an ultrasonic liquid processor for $45 \mathrm{~s}$ at $20 \mathrm{~W}$ to obtain homogeneously dispersed ormosil colloids. $250 \mathrm{~mL}$ portions of the ormosil colloidal solution were then spin-coated onto clean glass substrates $(1 \times 2 \mathrm{~cm})$ at $3000 \mathrm{rpm}$ for $45 \mathrm{~s}$. Coatings were dried overnight at room temperature.

\subsection{Preparation of superhydrophilic patterns}

Superhydrophobic ormosil coatings were covered with cellulose acetate sheets which were previously hollowed at pre-defined areas using a $30 \mathrm{~W} \mathrm{CO}_{2}$ laser cutting system (Epilog Zing, US). Then, surfaces were exposed to UV/O for varying durations (5-60 min) at ambient temperature using a surface cleaning system (PSD-UV, Novascan Technologies, US). Power intensity is 8 and $30 \mathrm{~mW} \mathrm{~cm} \mathrm{~cm}^{-2}$ for $185 \mathrm{~nm}$ and $253.7 \mathrm{~nm}$ wavelengths, respectively.

\subsection{Protein and bacteria adsorption experiments}

Arrays of circular patterns ( $1 \mathrm{~mm}$ diameter) with varying degrees of wettability were generated on superhydrophobic surfaces by varying the UV/O exposure time (15, 30 and $60 \mathrm{~min})$. The asprepared microchips were then soaked into $1 \mathrm{mg} \mathrm{mL}^{-1}$ FITCBSA aqueous solution for $30 \mathrm{~s}$ and then gently washed with DI water to remove the non-adsorbed proteins. The proteinadsorbed microchip surfaces were then imaged using confocal microscopy. For bacteria adsorption studies, GFP (green fluorescent protein)-expressing $E$. coli with size of $2 \mu \mathrm{m}$ was proliferated in $\mathrm{LB}$ growth medium at $37^{\circ} \mathrm{C}$ under $\mathrm{CO}_{2}$ environment. Then, LB was centrifuged at $2000 \mathrm{rpm}$ for $5 \mathrm{~min}$ and bacteria cells were dispersed in PBS (phosphate buffer saline, $\mathrm{pH} \sim 7.4$ ) medium. Approximately $200 \mu \mathrm{L}$ of the bacteria suspension was rolled over the square-patterned surface and microdroplets of the suspension were formed on the wetted spots.

\subsection{High-throughput mixing experiment}

Two patterned surfaces with hydrophilic spots were prepared by treating with UV/O for $30 \mathrm{~min}$ and $60 \mathrm{~min}$, respectively. Colored droplet arrays were generated by individually dispensing dyed solutions (methylene blue and rhodamine 6G aqueous solutions) to the hydrophilic spots on two separate surfaces. Identical array sizes (a $4 \times 6$ array) were used. Patterned surfaces were aligned using a microstage. 30 min-treated surface was placed up-side-down on the top and 60 min-treated surface was placed on the bottom. Droplet arrays were contacted using the microstage. Each individual droplet on the top surface mixed with its counterpart on the bottom surface. No lateral mixing was observed between the droplets on the same surface. Most of the mixed solution remained on the bottom surface due to the gravity and higher degree of wettability of the circular patterns (i.e. longer $\mathrm{UV} / \mathrm{O}$ treatment time).

\subsection{Characterizations}

Surface morphology of the surfaces was analysed with scanning electron microscope (E-SEM, Quanta 200F, FEI) under high vacuum condition at $10 \mathrm{kV}$ after coating the surfaces with $6 \mathrm{~nm}$ thick gold/platinum layer. Chemical analysis of the surfaces was performed using X-ray photoelectron spectroscopy (Thermo Fisher Scientific, UK) with Al K- $\alpha$ X-ray gun $(12 \mathrm{kV}, 2.5 \mathrm{~mA}$, and spot size of $400 \mu \mathrm{m}$ ) and an electron take-off angle of $90^{\circ}$. Avantage software was used for peak identification and fitting. Static water contact angles (WCA) of the surfaces were measured with a contact angle meter (OCA 30, Dataphysics). $4 \mu \mathrm{L}$ of water droplets were used for the measurements which were analysed with Laplace-Young equation. Fluorescent images of the protein-adsorbed surfaces were taken with a confocal microscope (Model LSM 510, Zeiss, Germany) using $10 \times$ objectives. Argon laser was used for excitation at $488 \mathrm{~nm}$ and emission at $505 \mathrm{~nm}$ was collected. Droplet capture on superhydrophilic spots was recorded using a high-speed camera (Phantom Miro M310, Vision Research, US) at 2000 fps.

\section{Results and discussion}

Superhydrophobic coatings were prepared from ormosil hybrid nanostructures as introduced in our previous report ${ }^{39}$ and then patterned as superhydrophilic-superhydrophobic using a rapid and simple UV/O treatment step. Colloidal ormosil nanoparticles were synthesized via a template-free sol-gel method using methyltrimethoxysilane (MTMS) as the organosilane 
precursor and deposited onto glass substrates. SEM image of the as-prepared coating revealed the highly porous and phaseseparated ormosil structure with both nanometer and micrometer pores (Fig. 1a). The rough and porous structure combined with the low surface-energy methyl group led to the superhydrophobicity with a static WCA of $170^{\circ}$ (Fig. 1a inset). As-prepared superhydrophobic surfaces were treated using UV/ $\mathrm{O}$ treatment where hydrophobic organic moieties were gradually decomposed. Contact angle of the surfaces decreased with treatment. Applying $1 \mathrm{~h}-\mathrm{UV} / \mathrm{O}$ treatment, superhydrophilic surfaces with WCA of nearly $0^{\circ}$ were obtained (Fig. 1b inset). The surface morphology and porous structure of the treated coating remained unchanged (Fig. 1b).

X-Ray photoelectron spectroscopy (XPS) was used to investigate the changes in the chemical structure of the ormosil surface after UV/O treatment. Survey analysis showed that untreated ormosil surface structure was composed of approximately $37.3 \%$ oxygen, $20.6 \%$ carbon and $42.1 \%$ silicon revealing the organicinorganic hybrid structure of ormosil. It is well known that in the presence of oxygen, UV light (185 and $254 \mathrm{~nm}$ ) generates ozone and reactive atomic oxygen atoms which cleave surface-bound hydrocarbons and form hydrophilic hydroxyl (-OH) groups. ${ }^{\mathbf{4 0 4 1}}$ Accordingly, chemical composition of the 1 h-treated surface was calculated to be $51.9 \%$ oxygen, $6.3 \%$ carbon and $41.8 \%$ silicon due to the decomposition of surface methyl groups and oxygen enrichment on the surface (Fig. 1c). Fig. 1d shows the high resolution $\mathrm{C}$ 1s spectra of the untreated and $1 \mathrm{~h}-\mathrm{UV} / \mathrm{O}$ treated surface. Peaks at 284.5 and $285.8 \mathrm{eV}$ correspond to the $\mathrm{C}-\mathrm{H}$ bond of methyl groups and $\mathrm{C}-\mathrm{O}$ bonds of non-hydrolyzed methoxy $\left(-\mathrm{O}-\mathrm{CH}_{3}\right)$ groups, respectively. The decrease of both $\mathrm{C}-\mathrm{H}$ and (a)

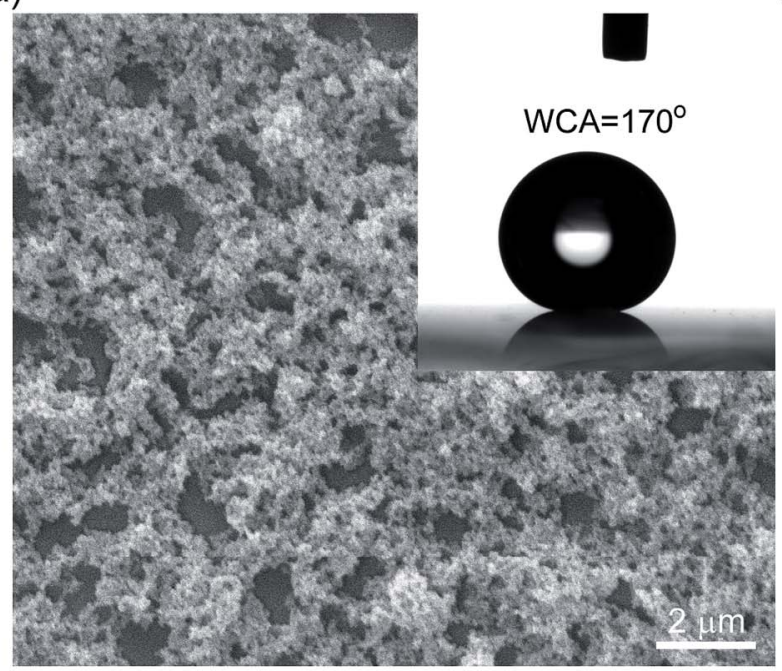

(C)

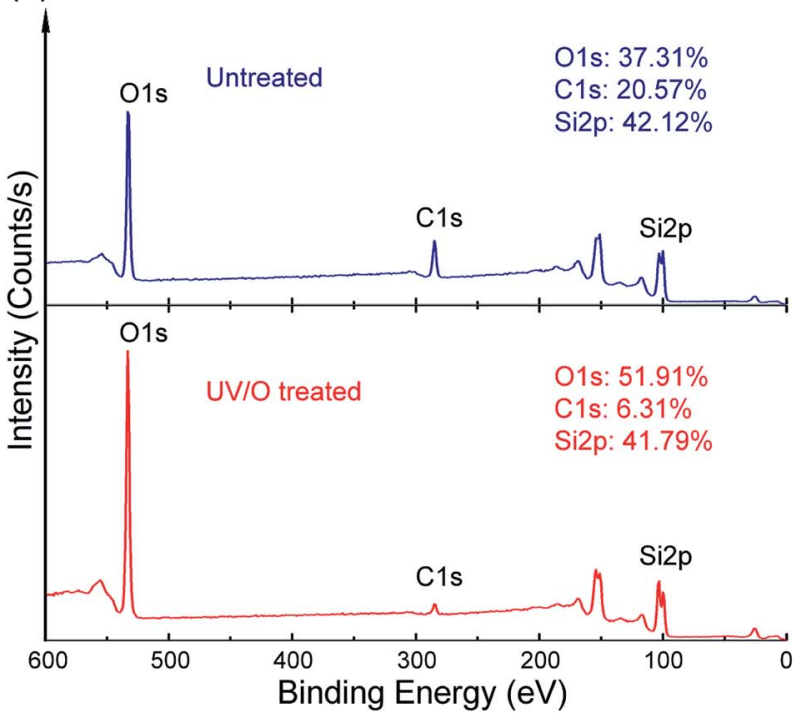

(b)

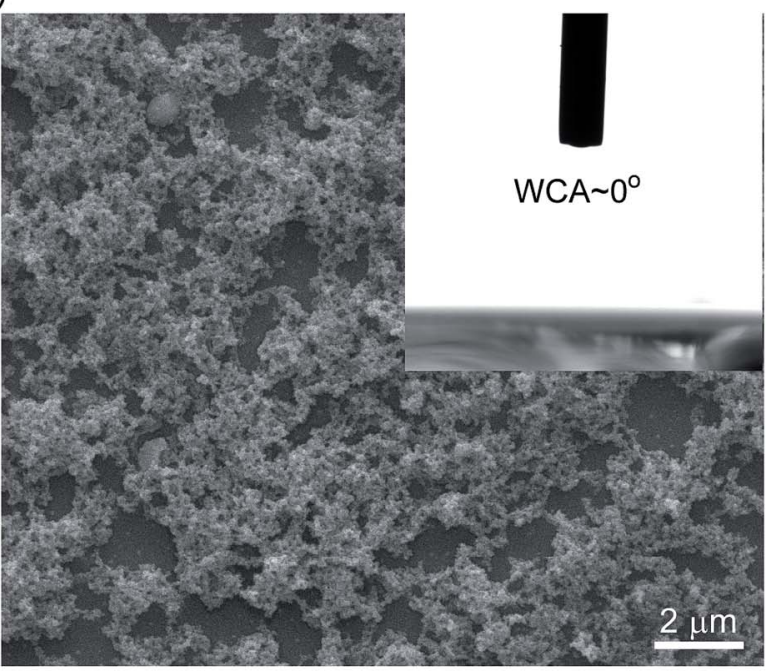

(d)

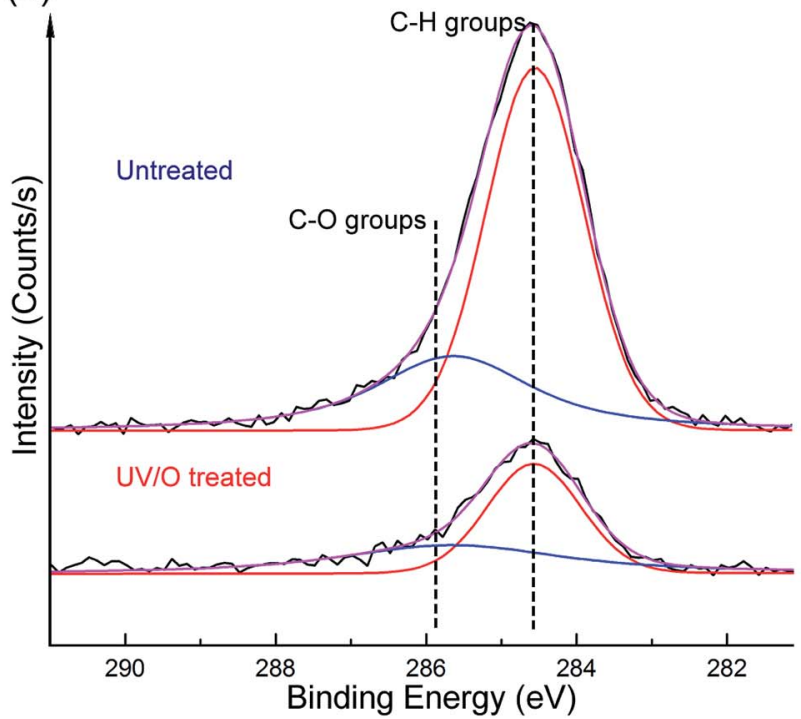

Fig. 1 (a) Scanning electron microscopy (SEM) image of the superhydrophobic ormosil surface with water droplet profile as the inset image. (b) SEM image of the $1 \mathrm{~h}-\mathrm{UV} / \mathrm{O}$-treated ormosil surface with completely spreading water as the inset image. (c) X-ray photoelectron spectroscopy (XPS) survey spectra of the ormosil surfaces before and after $1 \mathrm{~h}-\mathrm{UV} / \mathrm{O}$ treatment. (d) High-resolution $\mathrm{C} 1 \mathrm{~s}$ spectra of the ormosil surfaces before and after $1 \mathrm{~h}-U \mathrm{~V} / \mathrm{O}$ treatment. 
$\mathrm{C}-\mathrm{O}$ bond intensities upon treatment indicated that besides methyl groups, non-hydrolyzed methoxy groups were also decomposed.

Control of the degree of wettability in a wide range from superhydrophobic to superhydrophilic state is very important for various applications such as liquid transport, droplet handling, and biomolecular adsorption. ${ }^{15}$ We demonstrate the precise control of wettability of the ormosil coatings from superhydrophobic to superhydrophilic with WCA ranging from $170^{\circ}$ to $0^{\circ}$ by adjusting the UV/O treatment duration. WCA of the superhydrophobic ormosil coating decreased linearly with increasing UV/O exposure time. Extreme wettability $\left(\mathrm{WCA} \sim 0^{\circ}\right.$ ) was achieved after $\sim 45 \mathrm{~min}$ treatment (Fig. 2). The profile of water droplet changed from nearly spherical to hemispherical within $20 \mathrm{~min}$ on the treated surface, and then to a thin liquid film after 45-60 min (Fig. 2).

Masked UV/O illumination was utilized to create wetted micropatterns on the superhydrophobic ormosil coatings (Fig. 3a). As-prepared superhydrophobic coatings were treated with UV/O for 60 min through shadow masks placed on the coatings. Regions under the hollow sites of the mask became selectively modified by UV/O whereas remaining parts were well shielded. Replacement of methyl groups with hydroxyl groups on the UV/O-exposed areas increased the degree of wettability. On the other hand, chemical groups in the untreated regions remained unaffected leading to the isolation of superhydrophobic and superhydrophilic domains (Fig. 3a). Spherical water droplet on the untreated region indicated the conservation of the superhydrophobic property whereas colored solution spreading on the treated area confirmed the complete wetting behavior (Fig. 3b).

Well-defined superhydrophilic patterns with various size and geometries including square, stripe and circle were formed using different mask designs (Fig. 4). Multiplexed analysis can

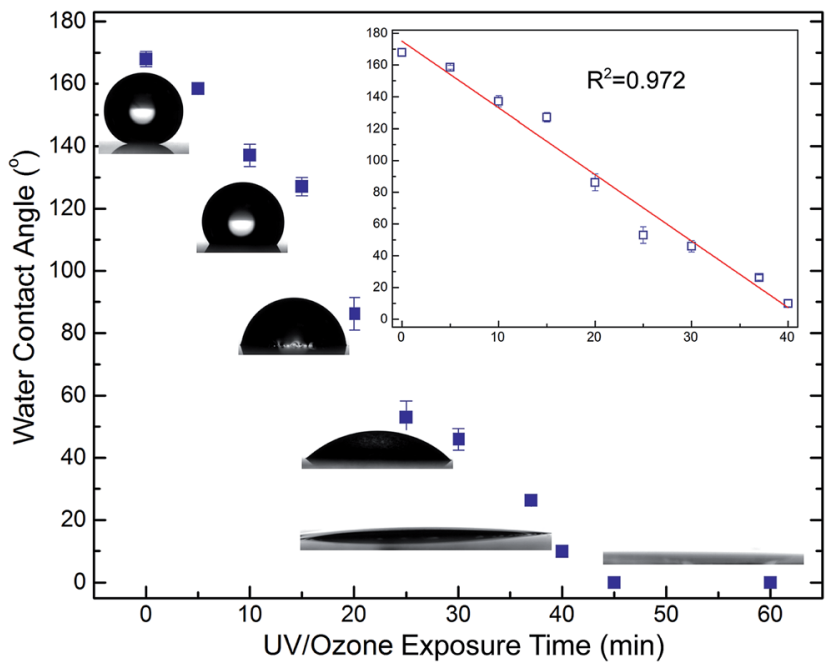

Fig. 2 Change in the water contact angles on UV/O-exposed ormosil surfaces depending on the treatment time. Inset images represent the droplet profiles captured for $0,10,20,30,40$ and 60 min treatment durations from top to bottom. Inset graph shows the linear fit for the decreasing behavior of WCA as a function of treatment time.
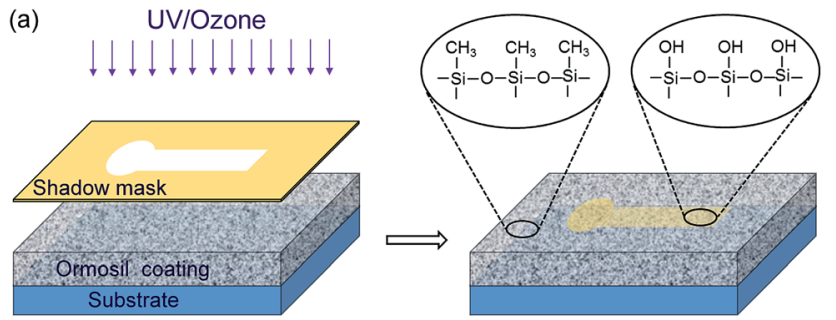

(b)

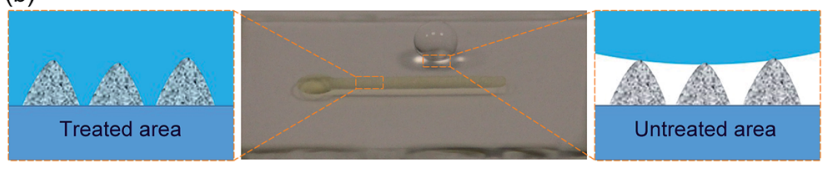

Fig. 3 (a) Schematic representation of UV/O treatment on superhydrophobic ormosil surfaces (left) and chemical groups on the treated and untreated areas (right). (b) Patterned ormosil surface with completely spreading FITC-BSA solution on the superhydrophilic area and spherical water droplet sitting on the superhydrophobic area. Corresponding schemes indicate the complete wetting and Cassie state non-wetting on the treated and untreated areas, respectively.

be performed on the patterned microchips since one can individually aliquot various aqueous components to distinct superhydrophilic spots (Fig. 4a). High density droplet microarrays can also be generated on the patterned surfaces by dipping them into aqueous media or by rolling a large droplet over the surface (Fig. 4c). Formation of droplet arrays was recorded using a high-speed camera at $2000 \mathrm{fps}$ on the patterned chip surface with wetted spots of $200 \mu \mathrm{m}$ diameter (Video SV1 and Fig. S1 in the ESI $\dagger$ ). Upon tilting the surface, distribution of small droplets to the individual spots from the
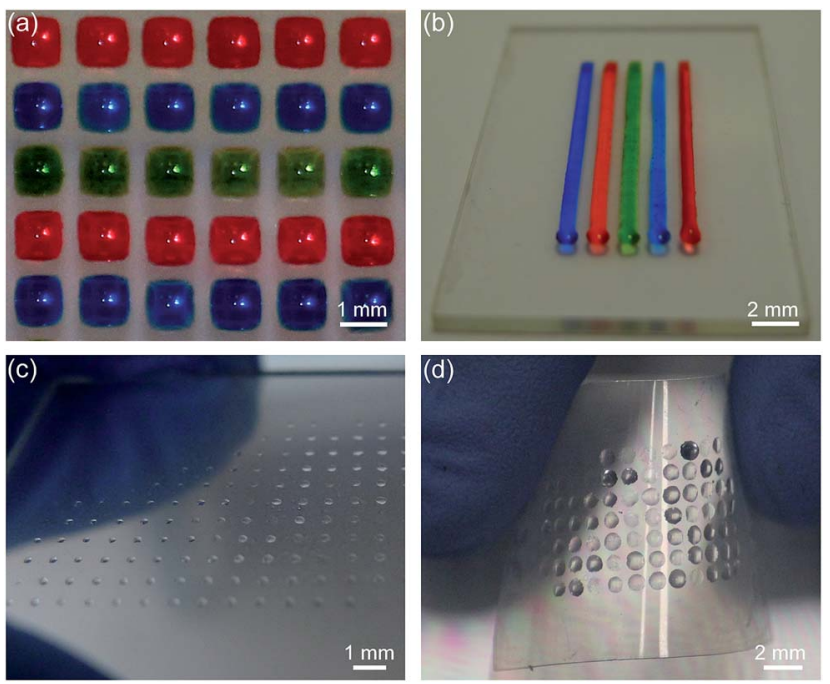

Fig. 4 (a) Ormosil surface with square-shaped super-wetted patterns holding different colored droplets (each pattern edge is $1 \mathrm{~mm}$ ). (b) Ormosil surface on glass substrate with colored aqueous solutions in stripe patterns with $1 \mathrm{~mm}$ width (blue dye is methylene blue, red dye is rhodamine $6 \mathrm{G}$, and green dye is mixture of acridine orange and methylene blue). (c) High-density droplet array in circle patterns with $200 \mu \mathrm{m}$ diameter. (d) Patterned ormosil surface on a bent cellulose acetate sheet and droplet array on circular superhydrophilic patterns. 
rolling large droplet was clearly observed. Low surface-energy beyond the boundary of the high surface-energy patterns induced the separation of small droplets. ${ }^{16}$ Furthermore, superhydrophilic and superhydrophobic patterns remained intact under bending impact owing to the flexibility of hybrid ormosil that preserves its structure under mechanical stress (Fig. 4d). ${ }^{42}$ Since time-dependent stability of superhydrophilic/ superhydrophobic patterns is desired for long-life analysis platforms, we examined contact angles of micropatterned ormosil chips after 5 month-storage at ambient conditions. After 5 months, we have not observed a remarkable change in WCA confirming that patterned chip surfaces conserved their initial wettability performance during the storage period (Fig. S2 in the ESI $\dagger$ ).

In order to demonstrate high-throughput biomedical screening potential, patterned surfaces were exploited for FITC-BSA adsorption. Surfaces exhibited fluorescent signal on the patterns with no signal on the untreated regions revealing the selective adsorption on the wetted patterns (Fig. 5a-c). Interestingly, fluorescence intensity, i.e. concentration of FITC-BSA increased with the increasing UV/O treatment time which was correlated with increase of degree of wettability (Fig. 5d and e). In addition, diameter of the fluorescent spots was slightly higher for $30 \mathrm{~min}$ - and $60 \mathrm{~min}$-treated patterns compared to the 15 min-treated counterpart (Fig. 5a-c). These findings showed that the adsorbed BSA density as well as its spatial distribution can be controlled by adjusting the degree of wettability from hydrophilic to superhydrophilic. Furthermore, we demonstrated the adsorption of GFP-expressing $E$. coli on the square-shape superhydrophilic patterns of ormosil films. Fluorescent signal of GFP which was observed only in the superhydrophilic patterns shows the good confinement of the bacteria cells in isolated wetted areas (Fig. S3 in the ESI $\dagger$ ). (d)

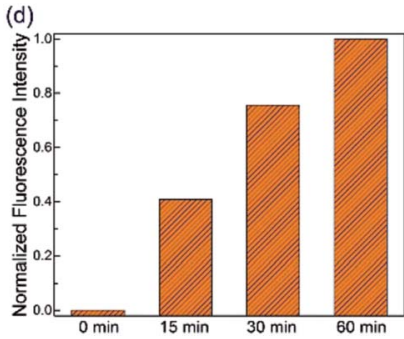

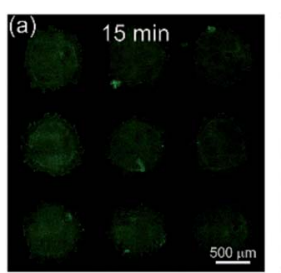

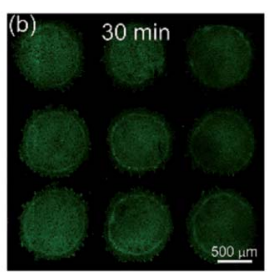

(e)

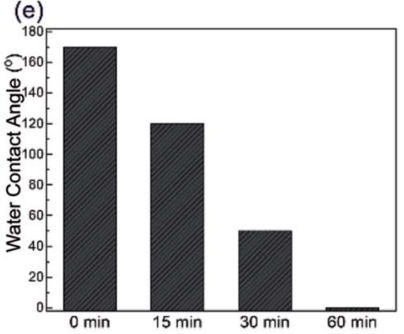

Fig. 5 Fluorescent images of the BSA-adsorbed patterned surfaces prepared via UV/O treatment for (a) $15 \mathrm{~min}$, (b) $30 \mathrm{~min}$ and (c) $60 \mathrm{~min}$. Fluorescent signals denoted as green correspond to the emission of FITC which is conjugated with BSA whereas the black background corresponds to the superhydrophobic regions with no fluorescent signal. (d) Normalized fluorescence intensities of the wetted patterns with respect to the UV/O treatment time. (e) WCA values on the treated ormosil surfaces with respect to the UV/O exposure time.
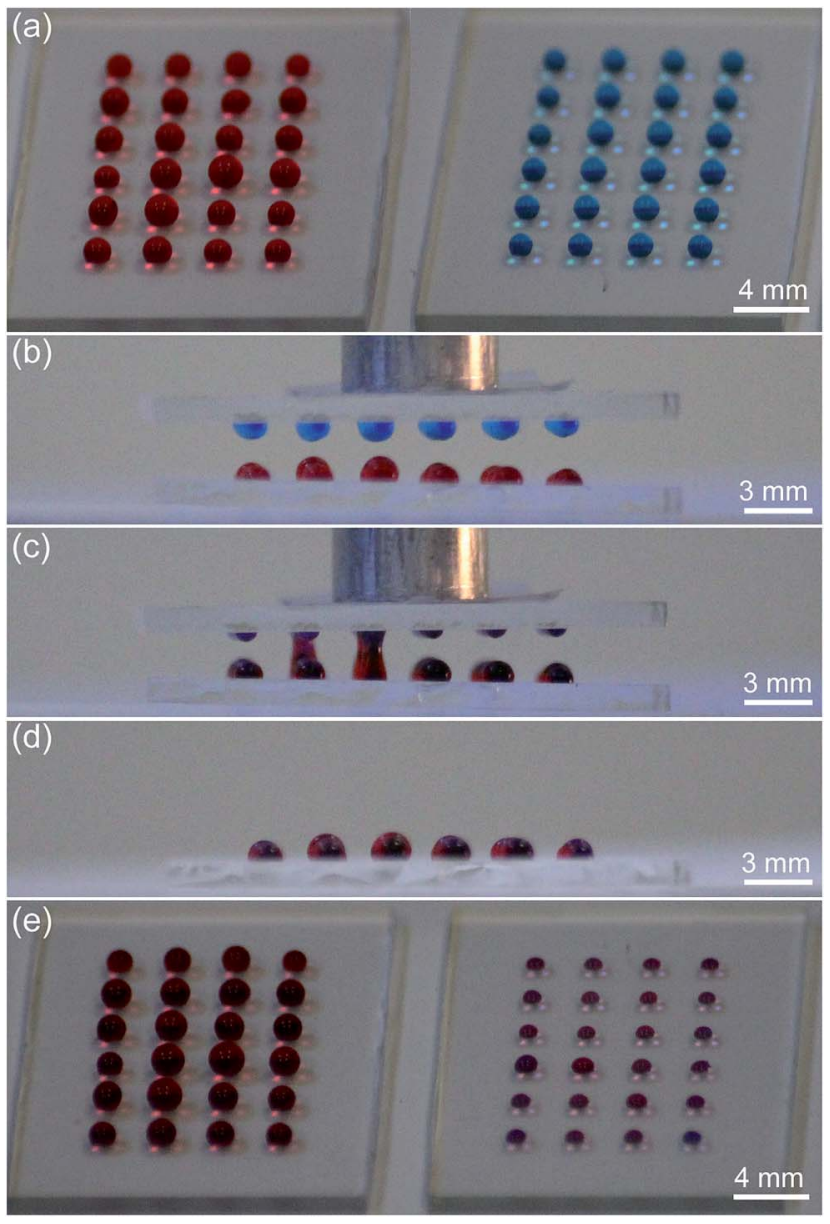

Fig. 6 High-throughput mixing of individual droplets on the patterned ormosil surfaces. (a) Colored droplet arrays (blue dye is methylene blue and red dye is rhodamine 6G) on two separate surfaces. (b) Patterned surfaces aligned using a microstage. Droplet arrays (c) during the contact and (d) after the contact. (e) Arrays of mixed droplets.

High-throughput mixing was also demonstrated on the patterned surfaces by using droplet arrays produced with two different contents which can be exploited for drug screening applications (Fig. 6).

\section{Conclusions}

In conclusion, we have demonstrated superhydrophilic patterning on superhydrophobic ormosil surfaces. Ormosil is an intrinsically superhydrophobic inorganic-organic hybrid structure and can be applied as functional nanoporous coating on different substrates including glass, silicon, and polymer. UV/O treatment on the selected areas of the ormosil surface leads to the formation of very stable superhydrophilic patterns without changing the surface morphology. Arrays of droplets can be formed in desired size and shape on both flexible and rigid materials. Super-wetted patterns has the capability of adsorbing any water-soluble materials which can be used for highthroughput molecular screening. Patterned ormosil surfaces with their simple production, robustness and versatility present 
a value added for practical applications in biomedical and chemical analysis.

\section{Acknowledgements}

We would like to thank Prof. Urartu Seker and Tolga Tarkan Olmez for providing bacteria cell cultures and Ziya Isiksacan for his help in high-speed recordings. This work is supported by TÜBİTAK under the Project No. 111T696. P. B. and U. T. were supported by TÜBİTAK-BIDEB graduate fellowship. C. E. acknowledges partial support from EU FP7 Marie Curie Career Integration Grant No. 322019.

\section{Reference}

1 A. Lafuma and D. Quere, Nat. Mater., 2003, 2, 457-460.

2 S. Nishimoto and B. Bhushan, RSC Adv., 2013, 3, 671-690.

3 L. Zhai, M. Berg, F. Cebeci, Y. Kim, J. Milwid, M. Rubner and

R. Cohen, Nano Lett., 2006, 6, 1213-1217.

4 R. Garrod, L. Harris, W. Schofield, J. McGettrick, L. Ward,

D. Teare and J. Badyal, Langmuir, 2007, 23, 689-693.

5 H. Bai, L. Wang, J. Ju, R. Sun, Y. Zheng and L. Jiang, Adv. Mater., 2014, 26, 5025-5030.

6 L. Zhang, J. Wu, M. Hedhili, X. Yang and P. Wang, J. Mater. Chem. A, 2015, 3, 2844-2852.

7 N. Oliveira, A. Neto, W. Song and J. Mano, Appl. Phys. Express, 2010, 3, 085205.

8 K. Choi, A. H. C. Ng, R. Fobel and A. R. Wheeler, Annu. Rev. Anal. Chem., 2012, 5, 413-440.

9 P. Lam, K. Wynne and G. Wnek, Langmuir, 2002, 18, 948-951.

10 I. You, N. Yun and H. Lee, ChemPhysChem, 2013, 14, 471481.

11 S. Xing, J. Jiang and T. Pan, Lab Chip, 2013, 13, 1937-1947.

12 J. Songok, M. Tuominen, H. Teisala, J. Haapanen, J. Makela, J. Kuusipalo and M. Toivakka, ACS Appl. Mater. Interfaces, 2014, 6, 20060-20066.

13 E. Ueda and P. Levkin, Adv. Mater., 2013, 25, 1234-1247.

14 E. Gogolides, K. Ellinas and A. Tserepi, Microelectron. Eng., 2015, 132, 135-155.

15 A. Neto, C. Custodio, W. Song and J. Mano, Soft Matter, 2011, 7, 4147-4151.

16 E. Ueda, F. Geyer, V. Nedashkivska and P. Levkin, Lab Chip, 2012, 12, 5218-5224.

17 A. Neto, C. Correia, C. Custodio and J. Mano, Adv. Funct. Mater., 2014, 24, 5096-5103.

18 F. Geyer, E. Ueda, U. Liebel, N. Grau and P. Levkin, Angew. Chem., Int. Ed., 2011, 50, 8424-8427.

19 H. Lim, J. Han, D. Kwak, M. Jin and K. Cho, J. Am. Chem. Soc., 2006, 128, 14458-14459.
20 S. Pastine, D. Okawa, B. Kessler, M. Rolandi, M. Llorente, A. Zettl and J. Frechet, J. Am. Chem. Soc., 2008, 130, 42384239.

21 D. Zahner, J. Abagat, F. Svec, J. Frechet and P. Levkin, Adv. Mater., 2011, 23, 3030-3034.

22 W. Feng, L. Li, E. Ueda, J. Li, S. Heissler, A. Welle, O. Trapp and P. Levkin, Adv. Mater. Interfaces, 2014, 1, 1400269.

23 X. Li, J. Tian, T. Nguyen and W. Shen, Anal. Chem., 2008, 80, 9131-9134.

24 V. Jokinen, L. Sainiemi and S. Franssila, Adv. Mater., 2008, 20, 3453-3456.

25 S. Kobaku, A. Kota, D. Lee, J. Mabry and A. Tuteja, Angew. Chem., Int. Ed., 2012, 51, 10109-10113.

26 J. Li, E. Ueda, A. Nallapaneni, L. Li and P. Levkin, Langmuir, 2012, 28, 8286-8291.

27 U. Manna, A. Broderick and D. Lynn, Adv. Mater., 2012, 24, 4291-4295.

28 D. Tian, Y. Song and L. Jiang, Chem. Soc. Rev., 2013, 42, 51845209.

29 T. Schutzius, I. Bayer, G. Jursich, A. Das and C. Megaridis, Nanoscale, 2012, 4, 5378-5385.

30 K. Tadanaga, J. Morinaga, A. Matsuda and T. Minami, Chem. Mater., 2000, 12, 590-592.

31 X. Zhang, H. Kono, Z. Liu, S. Nishimoto, D. Tryk, T. Murakami, H. Sakai, M. Abe and A. Fujishima, Chem. Commun., 2007, 4949-4951.

32 J. Huang, Y. Lai, L. Wang, S. Li, M. Ge, K. Zhang, H. Fuchs and L. Chi, J. Mater. Chem. A, 2014, 2, 18531-18538.

33 G. Soliveri, R. Annunziata, S. Ardizzone, G. Cappelletti and D. Meroni, J. Phys. Chem. C, 2012, 116, 26405-26413.

34 K. Nakata, S. Nishimoto, Y. Yuda, T. Ochiai, T. Murakami and A. Fujishima, Langmuir, 2010, 26, 11628-11630.

35 S. Nishimotoa, M. Becchakua, Y. Kameshimaa, Y. Shirosakib, S. Hayakawab, A. Osakab and M. Miyakea, Thin Solid Films, 2014, 558, 221-226.

36 H. Hillborg, N. Tomczak, A. Olah, H. Schonherr and G. Vancso, Langmuir, 2004, 20, 785-794.

37 Y. Li, Z. Wang, L. Ou and H. Yu, Anal. Chem., 2007, 79, 426433.

38 P. van Midwoud, A. Janse, M. Merema, G. Groothuis and E. Verpoorte, Anal. Chem., 2012, 84, 3938-3944.

39 H. Budunoglu, A. Yildirim, M. Guler and M. Bayindir, ACS Appl. Mater. Interfaces, 2011, 3, 539-545.

40 H. Kim, C. Kreller, K. Tran, V. Sisodiya, S. Angelos, G. Wallraff, S. Swanson and R. Miller, Chem. Mater., 2004, 16, 4267-4272.

$41 \mathrm{~K}$. Efimenko, W. Wallace and J. Genzer, J. Colloid Interface Sci., 2002, 254, 306-315.

42 H. Budunoglu, A. Yildirim and M. Bayindir, J. Mater. Chem., 2012, 22, 9671-9677. 